\title{
Lightness Pretreatment with Pedestrian Detection Studies Using CH\&K Algorithm for a Night Environment
}

\author{
Jun-Gyu Parkand Dae-Seong Kang \\ Dong-A University, Dept. of Electronics Engineering, 37, Nakdong-daero \\ 550beon-gil, Saha-gu, Busan,Korea \\ jungyuyo@naver.com
}

\begin{abstract}
This paper presents a real-time pedestrian detection algorithm for a night environment. By first converting the nighttime image data to the $L * a * b *$ color space, it can be extracted in the area $L *$ with robust noise reduction and contrast adjustment. This data is used to generate pre-data through image subtraction. A background image is generated using the data, and the Cascade Histogram of Oriented Gradient \&Kalman filter $(\mathrm{CH} \& \mathrm{~K})$ algorithm is proposed to track the movement of pedestrians. In addition, pre-processing algorithms and the proposed algorithm can replace Histograms of Oriented Gradients (HOGs) with rather heavy computations, and sensitive Haar-like features in the night can be used for real-time pedestrian detection and brightness.
\end{abstract}

Keywords: $L^{*} a^{*} b^{*}, C H \& K$ (Cascade Histogram of Oriented Gradient \&Kalman filter), Histograms of oriented gradient (HOGs), Haar-like features, Cascade

\section{Introduction}

Due to the recent increase in installed CCTVs and vehicle black boxes, these have been used as important evidence for solving various incidents. However, the majority of incident types occur more frequently in nighttime environments [1]. CCTV and general personal cameras, as well as black boxes, are very vulnerable at night or indoors where there is little or no light. Night CCTV data include many shadows and lighting, and image identification can be very difficult.

In this paper, a pedestrian tracking program that works even at night is proposed. Existing methods include the conventional pedestrian tracking method to track and analyze the human silhouette characteristic parameters [2]; using the image from the color information of the video by analyzing the division and outlining how to connect only similar colors to complete the blob [3]; the learning and classification algorithms Support Vector Machine (SVM) [4-5].Kalman Filtering Algorithm Based-on tracking System [67]. Expensive infrared cameras for night vision image are used for the improvement of existing methods. There is a method using a visible spectrum range camera, and there is a method using a camera installed to illuminate the installed place. However, the methods still used in camera applications have practical limitations to applications and research. After removal of the same c-based language to solve the problems of the $\mathrm{L}^{*} \mathrm{a} * \mathrm{~b} *$ color space, the image noise is reduced through the process, improving nighttime pedestrian data. In addition, the movement of pedestrians can be tracked through a Kalman filter using a cascade HOG learning pedestrian detector. 


\section{Related Theory}

\subsection{Lab Color Space}

Unlike the RGB and CMYK color models, Lab color is designed to approximate human vision. It aspires to perceptual uniformity, and its L component closely matches human perception of lightness, although it does not take the Helmholtz-Kohlrausch effect into account. Thus, it can be used to make accurate color balance corrections by modifying output curves in the a and $\mathrm{b}$ components or to adjust the lightness contrast using the L component. In RGB or CMYK spaces, which model the output of physical devices rather than human visual perception, these transformations can be done only with the help of appropriate blend modes in the editing application.

Because Lab space is much larger than the gamut of computer displays, printers, or even human vision, a bitmap image represented as Lab requires more data per pixel to obtain the same precision as an RGB or CMYK bitmap. In the 1990s, when computer hardware and software were limited to storing and manipulating mostly 8-bit/channel bitmaps, converting an RGB image to Lab and back was a very lossy operation. With 16bit/channel and floating-point support now common, the loss due to quantization is negligible [8].

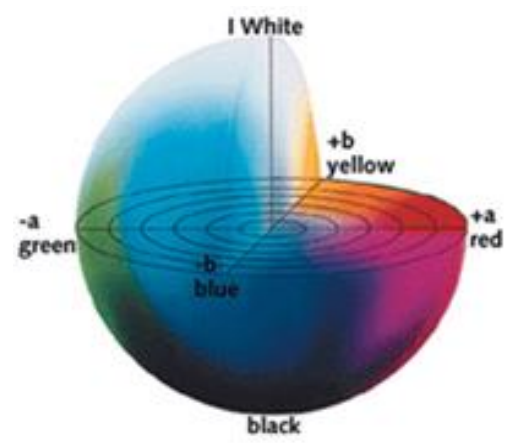

Figure 1. $L^{\star} A^{\star} B$ Color Space Model. (Cle Lab 1976)

The Lab color space is represented in Figure 1. The three coordinates of CLE LAB represent the lightness of the color $\left(\mathrm{L}^{*}=0\right.$ indicates black and $\mathrm{L}^{*}=100$ indicates diffuse white; specular white may be higher), its position between red/magenta and green (for $\mathrm{a}^{*}$, negative values indicate green, while positive values indicate magenta), and its position between yellow and blue (for $\mathrm{b}^{*}$, negative values indicate blue, and positive values indicate yellow). The asterisks $\left(^{*}\right)$ after $\mathrm{L}, \mathrm{a}$, and $\mathrm{b}$ are pronounced star and are part of the full name, since they represent $\mathrm{L}^{*}, \mathrm{a}^{*}$ and $\mathrm{b}^{*}$, to distinguish them from Hunter's $\mathrm{L}$, a, and $\mathrm{b}$, described below. It is possible to calculate the $\mathrm{L} * \mathrm{a} * \mathrm{~b} *$ data in the following equations (1) to (4). Equation (1) is a process of converting the RGB color coordinate space into the XYZ color coordinates. Equations (2), (3), and (4) are obtained from the formula $X(1), Y$, which is a formula for each of the information items assigned to $\mathrm{L}^{*} \mathrm{a} \mathrm{b}^{*}$ data for the $\mathrm{Z}$ value [9-10].

$$
\begin{gathered}
\left|\begin{array}{l}
X \\
Y
\end{array}\right|=\left|\begin{array}{lll}
0.43 & 0.34 & 0.18 \\
0.21 & 0.71 & 0.72 \\
0.02 & 0.13 & 0.94
\end{array}\right| \times\left|\begin{array}{l}
R \\
G \\
B
\end{array}\right| \\
L^{*}=116 \times f\left(\frac{Y}{Y_{n}}\right)-16 \\
a^{*}=500 \times\left[f\left(\frac{X}{X_{n}}\right)-f\left(\frac{Y}{Y_{n}}\right)\right]
\end{gathered}
$$




$$
\begin{gathered}
b^{*}=200 \times\left[f\left(\frac{Y}{Y_{n}}\right)-f\left(\frac{Z}{Z_{n}}\right)\right] \\
\text { where } f(t)=\left\{\begin{array}{l}
t^{\frac{1}{3}} \quad \text { if } t>\left(\frac{6}{29}\right)^{3} \\
\frac{1}{3}\left(\frac{29}{6}\right)^{2} t+\frac{4}{29} \text { otherwise }
\end{array}\right.
\end{gathered}
$$

$X_{n}, Y_{n}, Z_{n}$ Are the tristimulus values at the White point of CLE; $\mathrm{n}$ lies in the normalized value.

In this paper, to enable pedestrian tracking in a dark area, the $\mathrm{L}^{*}, \mathrm{a} *, \mathrm{~b} *$ color space was used for areas of lightness. Figure 2 compares examples of histogram smoothing pictures that are widely used in such a manner as to compensate for the lighting area lightness in the proposed model.

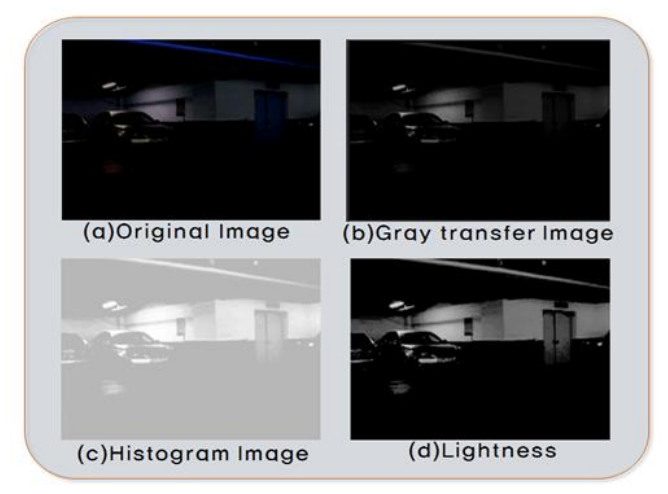

\section{Figure 2.Comparison of Histogram-Equalized and Lightness Area Methods}

Figure 2 (a) is an original image taken at night, (b) is a gray image conversion image, (c) is the transformed image histogram equalization method compared to the proposed method, and (d) is a detection area using lightness of the Lab color space to render the image. Figure 3 (c) is a pedestrian area; a motion is observed, which casts a dark shadow. However, there is overexposure to the influence of ambient light noise. While (d) in Figure 3 can be identified even in a dark area and the pedestrian can be seen, the results are compared to the removal of the noise exposure in (c). Therefore, it is possible to remove the appropriate pre-processing operation in the background.

\subsection{Cascade}

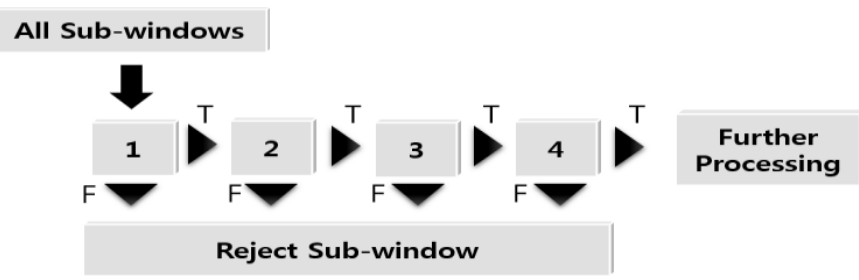

Figure 3. Cascade Classifier

The term cascade refers to the use of multiple detectors sequentially applied to the first. A strong detector takes a long time, but it may significantly increase the overall detection rate due only to the candidate having passed the initial simple detectors. However, in order to increase the detection performance, it requires a long learning process and a large 
amount of sample data. When learning, a large amount of data can result in good performance.

\subsection{Hog}

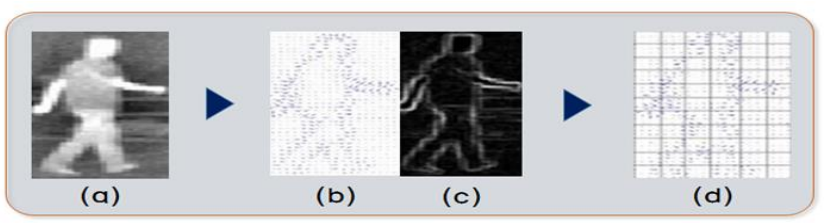

Figure 4.Detected Pedestrian Using Hog Algorithm

HOG is characterized by the direction of the brightness histogram of the distribution of the local region. The target area cells of a predetermined size are divided, and the edge gradient magnitude at each cell is connected in the series vector histogram bin value of those obtained after a histogram for pixels in the direction of a certain value or more. In other words, HOG can be seen in the direction of the template histogram of the edge. Under the influence of illumination, it is a less robust algorithm that is characterized by a geometric change in the local area. However, the computational burden is large, and the target detection speed is slow, which are considerable drawbacks. In Figure 4, (a) is a rough image of a pretreatment and walker, (b) is the gradient orientation, (c) is the gradient magnitude, and (d) illustrates a HOG[9]

\section{Proposed Method}

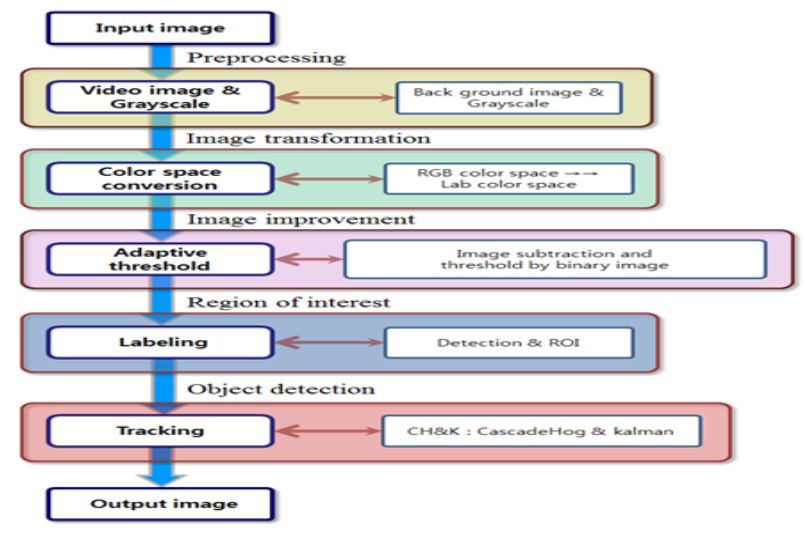

Figure 5. The Proposed Algorithm

\subsection{Color Space Conversion}

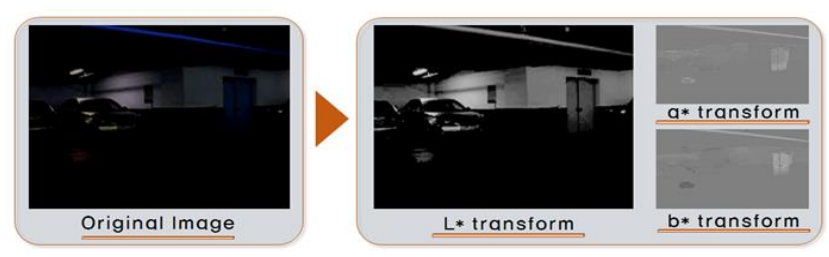

Figure 6. Lab Transform

This figure is converted into the $\mathrm{L} * \mathrm{a} * \mathrm{~b} *$ color space of the image in order to enable tracking of the pedestrian in a dark area. It can be seen that the original image is dark, but 
the car is parked and the contact position accurately shows the $\mathrm{L}^{*}$. In the screen area compared to the original image used in this paper, the object is well distinguished.

Figure 7 is an $\mathrm{L} * \mathrm{a} * \mathrm{~b} *$ image applied to pretreatment data.

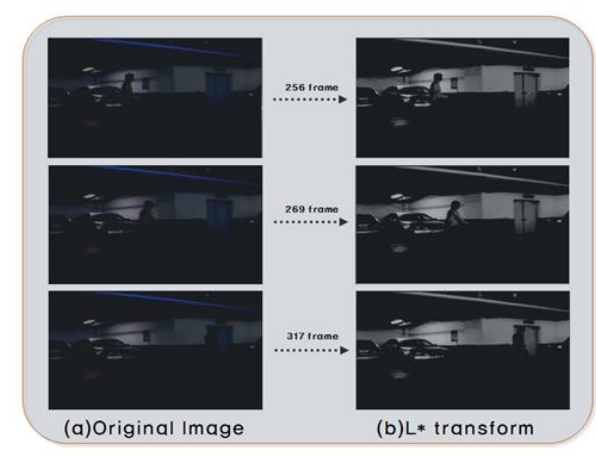

Figure 7. Lab Transform Image

The difference operation is the same $\mathrm{x}$ as that prior to the time the pixel data as a result of the difference of the current time, as well as the pixel brightness on the y-axis. First, the difference between the gray values in the coordinate conversion, and as a gray image, is determined. The difference value is applied to a predetermined threshold to represent a binary image of the 0 and 255. The foreground object candidate area is in the 255-pixel image, and the background pixel is read as zero. The threshold is set at 25. An advantage of the difference operation is the calculation speed.

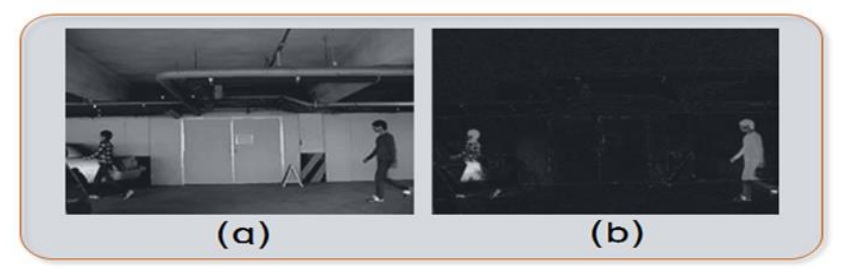

Figure 8. Gray-Image and Image Subtraction Result

A much smaller amount of calculation is achieved in the foreground and background separation algorithm than with any other method. With the appropriate correction method with arithmetic computation car, the Gaussian Mixture Model (GMM) does not need to be used extensively (Figure 8), and as in theory, only the moving objects remain by subtracting the video background from the image. However, in practice, this is exposed to a moving object, a shadow of the object, light noise, reflected noise, and noise from a number of non-pedestrian sources. In particular, the image data of the nighttime noise incidence is much more difficult.

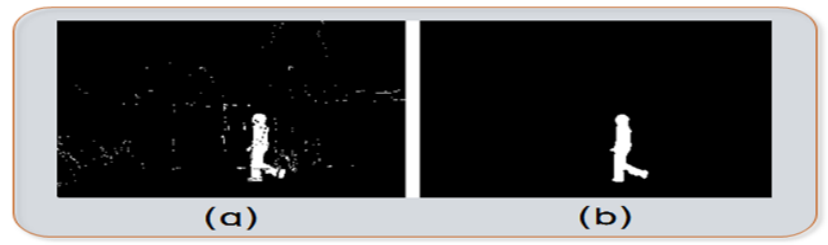

Figure 9. Closing Operation of the Morphological Image

As shown in Figure 8, with the theoretical consideration only when one tries to present an image, image subtraction of moving object detection in the background image is 
obtained. However, actually, the shadow of the object is also detected as a moving object image as well as reflected noise, lighting, etc. There are numerous noises mixed with a significant amount of noise. This is done in order to remove the closing operation of the expansion and erosion operations in morphology. Figure 9 confirms the results of the closing operation of the morphology.

Figure 9 is a processing result of the input image 1. (a) is a pedestrian silhouette and noise prior to image morphology caught. (b) Shows the resulting image after the I and performance the closing operation of the morphology.

Figure10. Closure morphology is the result of a calculation performed on the image with multiple pedestrian movements. It can be seen that the noise in the non-pedestrian millet is removed.

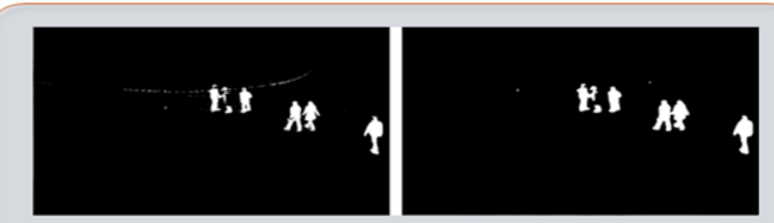

(a) (b)

Figure 10.Closing Operation ofthe Morphological Image 2

\section{Experimental Results}
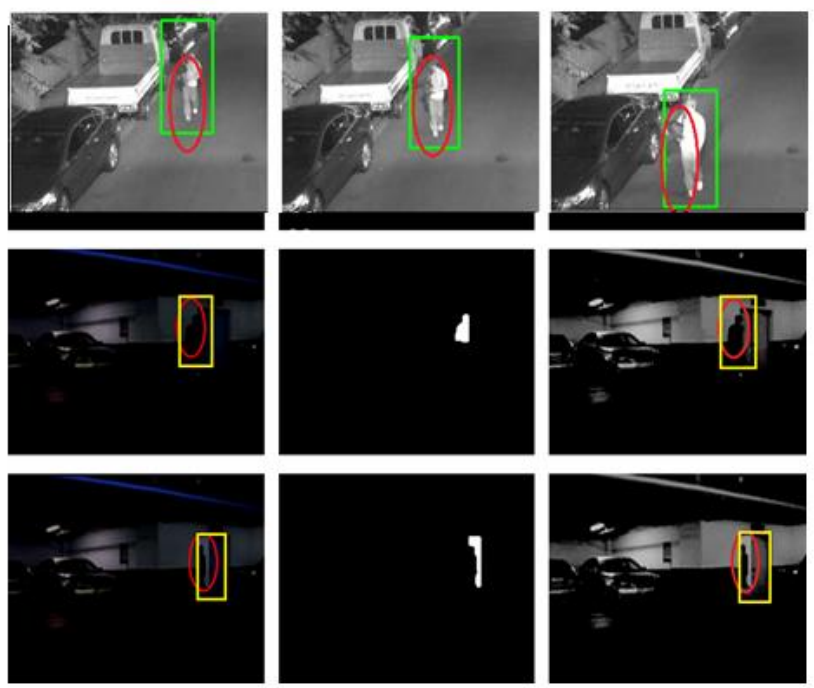

Figure 11. CH\& K Result

In night environments such as that seen in Figure 11, where the light is almost not used as a cellar $\mathrm{CH} \& \mathrm{~K}$, the proposed algorithm can detect a pedestrian. An example of the pedestrian information algorithm has been the learning algorithm Cascade HOG pedestrian detection, where detection speed and accuracy were increased by using it together with the Kalman algorithm.

\section{Conclusion}

The Haar-feature detection algorithm based on the brightness feature did not detect pedestrians in a night image, and the HOG algorithm to accurately detect pedestrians was not successful because of the impact on the surrounding light. However, pedestrian detection using the $\mathrm{CH} \& \mathrm{~K}$ pretreatment methods and algorithms proposed in this paper 
could achieve the same results as depicted in Figure 11. The $\mathrm{CH} \& \mathrm{~K}$ algorithm proposed in the paper for learning a large amount of data initially to increase the detection performance, then accuracy, of Cascade and rapid object recognition has the advantage of enabling the detection of the object in the image using a lower speed HOG. Adding the Kalman filter to predict the path of a pedestrian as well as the speed was faster and more accurate than the conventional HOG algorithm implementing a high pedestrian detection algorithm.

The average throughput rate is as shown in Figure 11, where the 23-frame $640 \mathrm{X} 480$ image size was used.The next goal is to detect a pedestrian in multi-night imaging and to study pedestrian operation speed that does not slow down in the overlap

\section{Acknowledgment}

This research was supported by Basic Science Research Program through the National Research Foundation of Korea (NRF) funded by the Ministry of Education, Science and Technology (2011-0011735)

\section{References}

[1] S. -Y. Jeong and D. -K.Byeon, "Research for improving the detecting pedestrian with Night Vision", Journal of The Institute of Electronics and Information Engineers, vol. 33, no. 1,(2010), pp. 1362-1364.

[2] W. Bing-bing, C.Zhi-xin, W.Jia and Z.Liquan,"Pedestrian detection based on the combination of HOG and background subtraction method",IEEE, TMEE,(2011), pp. 527-531.

[3] R.Lienhart and J.Maydt,“An Extended-Set of Haar-like Features for Rapid Object Detection”, IEEE Conference on ICIP, vol.1, (2002), pp.900-903.

[4] I.P. Alonso, D.F. Llorca, M.A. Sotelo and L.M.Bergasa, "Combination of Feature Extraction Methods for SVM Pedestrian Detection”, IEEE, vol.8, (2007), pp.292-307.

[5] V.Majidnezhad and I.Kheidorov,"The SVM-Based Feature Reduction in Vocal Fold Pathology Diagnosis", Journal of IJFGCN vol. 6, no.1,(2013).

[6] M. Li, B. -L. Zhu, S. -Y. Wang, Y. -C. Zhang and H. -Y. Li,"Strong Tracking Unscented Kalman Filtering Algorithm Based-on SatelliteAttitude Determination System”, Journal of IJFGCN vol. 7, no.3,(2014), pp. 155-166.

[7] D. K.Arif, S. Widodo and E.Apriliani,"Construction of the Kalman Filter Algorithm on the Model Reduction", Journal of IJCA vol. 7, no.9,(2014), pp.257-270.

[8] "L*a*b* color space Information", www.wikipedia.org/wiki/Lab_color_space

[9] G. -G. Lee, M.Uzair, J. -Y. Yoon, J. -J. Kim and W. -Y. Kim, “Comparisons of Color Spaces for Shadow Elimination”, Journal of Korea Multimedia Society, vol. 11, no. 5, (2008), pp. 610-622.

[10] K.Bae, L.Mesicek and H.Ko,"A Study on an Effective Feature Selection Method Using Hog-Family Feature for Human Detection”, Journal of IJMUE, vol.9, no.12, (2014), pp. 203-214.

\section{Authors}

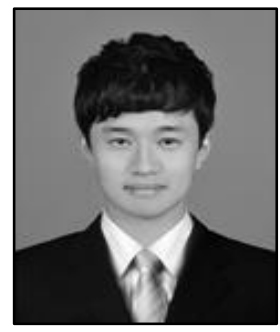

Jun-Gyu Park, He received a B.S. degree from Dong-A University, Busan, Korea, in 2014. He is in department of electronic engineering, Dong-A graduate university now. He research image processing.

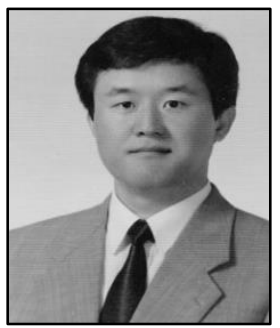

Dae-Seong Kang, He received a B.S. degree from Kyungpook National University, Daegu, Korea, in 1984, M.S. degree and D.Sc. degree in electrical engineering from Texas A\&M University, in 1991 and 1994, respectively. He is currentlyfull professor of the Department of Electronic Engineering, Dona-A University, Busan,Korea. His research interests are image processing and compression. 
International Journal of Smart Home

Vol. 9, No. 7 (2015) 